\title{
Resolution of Occlusive Carotid Artery Thrombus Treated with Anticoagulation as Demonstrated on Duplex Ultrasonography.
}

\author{
Laura Stein \\ Icahn School of Medicine at Mount Sinai \\ John W. Liang \\ Thomas Jefferson University \\ Jesse Weinberger \\ Icahn School of Medicine at Mount Sinai
}

Follow this and additional works at: https://jdc.jefferson.edu/neurologyfp

Part of the Neurology Commons

Let us know how access to this document benefits you

\section{Recommended Citation}

Stein, Laura; Liang, John W.; and Weinberger, Jesse, "Resolution of Occlusive Carotid Artery Thrombus Treated with Anticoagulation as Demonstrated on Duplex Ultrasonography." (2017). Department of Neurology Faculty Papers. Paper 149.

https://jdc.jefferson.edu/neurologyfp/149

This Article is brought to you for free and open access by the Jefferson Digital Commons. The Jefferson Digital Commons is a service of Thomas Jefferson University's Center for Teaching and Learning (CTL). The Commons is a showcase for Jefferson books and journals, peer-reviewed scholarly publications, unique historical collections from the University archives, and teaching tools. The Jefferson Digital Commons allows researchers and interested readers anywhere in the world to learn about and keep up to date with Jefferson scholarship. This article has been accepted for inclusion in Department of Neurology Faculty Papers by an authorized administrator of the Jefferson Digital Commons. For more information, please contact: JeffersonDigitalCommons@jefferson.edu. 
Title: Utility of carotid ultrasonography in management of an atypical high-vascular risk patient with recurrent calcified cerebral embolic stroke

John W. Liang M.D. ${ }^{1,2}$; Jesse Weinberger, M.D. ${ }^{1}$; Aaron Tansy, M.D. ${ }^{1}$

${ }^{1}$ Department of Neurology, Icahn School of Medicine at Mount Sinai, New York, NY 10128

${ }^{2}$ Department of Neurology, Divisions of Cerebrovascular Disease, Critical Care and Neurotrauma, Thomas Jefferson University, Philadelphia, Pennsylvania

\section{Corresponding Author:}

Aaron Tansy, M.D.

Assistant Professor of Neurology

Mount Sinai Icahn School of Medicine

Stroke Director, Mount Sinai Queens

One Gustave L. Levy Place

Box 1052

New York, NY 10029

t | 212.241.3870

f | 212.987.3301

e I aaron.tansy@mssm.edu

Grant support: None

Disclosures: None

Running title: Atypical calcified cerebral embolic stroke 


\section{Abstract}

Objective: To describe a case of recurrent calcified cerebral emboli related acute ischemic stroke and the diagnostic utility of plaque morphology characterization on carotid ultrasound

Background: Calcified cerebral emboli (CEE) is a rare cause of acute ischemic stroke (AIS). CEE-related AIS have been previously reported only in high vascular-risk patients such as those with severe carotid stenosis, widespread atheromatous disease, or cardiac valvular disease. CEE-related AIS from a carotid origin have not been reported in patients without carotid stenosis.

Case: A 69 year-old man with no known medical history presented with hemiparesis and aphasia, was found to have a curvilinear calcification in the left sylvian fissure on brain imaging, consistent with CEE. Two months later, he developed a second episode of CEE-related AIS. Standard work up, as well as advanced imaging with digital subtraction angiography, revealed no carotid stenosis or valvular disease. Carotid ultrasound demonstrated normal flow velocities but a left carotid heterogeneous plaque with multiple ulcerative craters and lucencies, suggestive of an active thromboembolic source.

Conclusion: To our knowledge, this is the first case reporting CEE-AIS from carotid origin in a patient with no carotid stenosis. Carotid ultrasound serves a diagnostic role in these patients. 


\section{Introduction}

Calcified cerebral emboli (CEE) are a potential, but relatively little known cause of acute ischemic stroke (AIS) ${ }^{1-7}$. CEE-related AIS presumably results when thromboembolic material dislodges from a proximal, calcified, unstable atherosclerotic lesion and travels distally to the cerebrovascular supply. Common arterial sources include: carotid stenosis, ${ }^{2,3}$ aortic valve, ${ }^{4}$ mitral valve,${ }^{5,7}$ and the brachiocephalic trunk. ${ }^{6}$ CEE-related AIS has been reported to occur both spontaneously ${ }^{8}$ and via provoked causes $^{3,9}$. Because of its infrequency, clinical information on CEE-related AIS is overall sparse within the current literature. One single-center study utilizing non-contrast CT (NCCT) estimated an approximate $3 \%$ prevalence rate in its stroke population; additionally, $27 \%$ of CEE on NCCT were misdiagnosed and $9 \%$ were overlooked on preliminary read by radiology residents. ${ }^{1}$ Furthermore, existing CEE-related literature has focused primarily only on high vascular-risk populations, the vast majority of which were due to high-grade arterial stenosis. ${ }^{2,8}$ We present a case of recurrent CEErelated, cryptogenic AIS in a patient who did not meet standard criteria for high vascular-risk status by routine and advanced cerebrovascular imaging methods, but ultimately was considered so through and underwent endovascular treatment for non-standard imaging criteria: lesion morphologic/qualitative findings on carotid ultrasound. This case highlights the potential importance of utilization of multimodal cerebrovascular imaging methods and integrative consideration of both standard and nonstandard imaging findings in management of CEE-related AIS.

\section{Case Report}

A 69 year-old man with no known medical history presented to our hospital network's primary stroke center with acute onset aphasia, left gaze deviation and right hemiparesis; (NIH stroke scale 18). NCCT revealed a curvilinear calcification in the left sylvian fissure (figure 1a). CT angiogram (CTA) revealed the calcification was non-occlusive and within a temporoparietal branch of the left middle cerebral artery; as well as bilateral carotid artery bifurcation atherosclerotic calcifications resulting in approximately $35 \%$ non-flow limiting stenosis. There was no calcification at the aortic arch. The patient was treated with intravenous alteplase, and managed subsequently per routine protocol. 24 hour posttreatment NCCT demonstrated fragmentation of the calcification with evolving infarct (figure 2a,b,c). Further evaluation including echocardiography, cardiac telemetry monitoring and outpatient long-term cardiac event monitoring revealed no definite thromboembolic source consistent with a cryptogenic event. Secondary stroke prevention optimal medical treatment including dual-agent antiplatelet therapy was initiated prior to discharge.

The patient presented again to the same center approximately two months later with recurrent isolated aphasia (NIH stroke scale 2). NCCT accounted for all previously seen calcified lesions and one newly seen calcified lesion within a previously unaffected vascular branch of the left middle cerebral artery (figure $2 \mathrm{~d}$ ). Treatment included intravenous alteplase, and managed again per routine protocol. MRI brain confirmed multiple small foci of acute infarction within the left middle cerebral arterial supply suggestive of an artery-to-artery thromboembolic phenomenon. The patient was subsequently 
transferred to our comprehensive stroke center for further care. He underwent digital subtraction angiography (DSA) which revealed bilateral ICA calcific atheroma with non-significant $<25 \%$ stenosis. Carotid ultrasound revealed normal flow patterns and velocities in both internal carotid arteries, a homogeneous appearing calcified plaque at the origin of the left ICA; as well as, slightly proximally, a heterogeneous plaque with multiple ulcerative craters and lucencies highly suggestive of an active, ruptured atherosclerotic lesion and thromboembolic source (figure 3a,b). Transesophageal echocardiography revealed no aortic arch plaques, left atrial appendage clots, or valvular calcifications (figure 4). Given these radiographic findings and recurrent AIS despite maximal medical therapy, the patient underwent treatment with endovascular stent placement for plaque stabilization and protection from future events.

\section{Discussion}

We report a case of recurrent CEE-related, cryptogenic AIS in a patient who did not meet standard criteria for high vascular-risk status, but through non-standard criteria found on multimodal cerebrovascular imaging was identified as such. Determination of an artery-to-artery thromboembolic source, initially not visualized with conventional and advanced imaging, was made ultimately through plaque morphology characterization uniquely appreciable with carotid ultrasound. Prior work in this laboratory has correlated ultrasound imaging of carotid plaque morphology with clinical events and pathological findings. ${ }^{10-12}$ This specialized diagnostic feature of carotid ultrasound highlights the potential utility and importance of integration and consideration of multimodal imaging techniques including ultrasound-aided plaque morphologic information when treating CEE-related AIS in patients with cryptogenic events and/or who may not meet standard criteria for high vascular-risk status. Importantly, although existing literature supports CEE-related AIS occurring in standard high-risk vascular risk patient populations ${ }^{2,3,6,8,13}$, this case report supports that it may also occur in nonstandard high-risk ones. Furthermore, although the impact of plaque morphology and its characterization in clinical management already have been described in typical high-risk vascular populations ${ }^{8,14,15}$, this report provides novel support that application of such non-routine carotid ultrasound morphologic imaging techniques provides potential unique utility as well in diagnosis and treatment for atypical high-risk vascular populations, particularly those with non-significant carotid stenosis like our patient had.

In summary, we describe a case of recurrent ipsilateral CEE-related, cryptogenic AIS in a patient ultimately determined to meet high vascular-risk status through nonstandard criteria obtained by carotid ultrasound. This case highlights the potential importance of utilization of multi-modal cerebrovascular imaging methods and integrative consideration of both standard and non-standard imaging findings especially ultrasound-aided plaque morphologic information in management of CEErelated AIS. Carotid ultrasound may play a role in the identification of the etiology of ischemic stroke in patients with cryptogenic stroke that do not have radiological findings of carotid stenosis by identifying large plaques with heterogeneous morphology in the common carotid artery and carotid bifurcation that are a potential embolic source. 


\section{FIGURE LEGENDS}

Figure 1: Non contrast brain CT with curvilinear calcification in the left sylvian fissure

Figure 2: $(A, B, C)$ : Non-contrast brain $C T$ 24-hours post intravenous alteplase with fragmentation of calcification (arrow) and development of parenchymal hypodensities (D) New calcified lesion in a previously unaffected vascular branch (arrowhead)

Figure 3: Ultrasound at origin of left internal and common carotid artery. Heterogenous low echodensity material (arrow) overlying plaque with underlying lucency (arrowhead) suggestive of an active, ruptured atherosclerotic lesion and thromboembolic source

Figure 4: Transesophageal echocardiogram of (A) aortic arch with no evidence of atheroma; (B) left atrial appendage with no thrombus, mid-esophageal view at 67 degrees; (C) non-calcified mitral valve, mid-esophageal view at 61 degrees; (D) non-calcified aortic valve, mid-esophageal view at 135 degrees 


\section{References}

1. Walker BS, Shah LM, Osborn AG. Calcified cerebral emboli, a "do not miss" imaging diagnosis: 22 new cases and review of the literature. AJNR Am J Neuroradiol. 2014;35:1515-1519

2. Christian BA, Kirzeder DJ, Boyd J, Laing J, Gash JR. Showered calcific emboli to the brain, the 'salted pretzel' sign, originating from the ipsilateral internal carotid artery causing acute cerebral infarction. Stroke; a journal of cerebral circulation. 2009;40:e319-321

3. Dandamudi VS, Thaler DE, Malek AM. Cerebral embolus following chiropractic manipulation in a patient with a calcified carotid artery. Journal of neuroimaging : official journal of the American Society of Neuroimaging. 2013;23:429-430

4. Rancurel G, Marelle L, Vincent D, Catala M, Arzimanoglou A, Vacheron A. Spontaneous calcific cerebral embolus from a calcific aortic stenosis in a middle cerebral artery infarct. Stroke; $a$ journal of cerebral circulation. 1989;20:691-693

5. Herskovitz M, Telman G, Carasso S, Symonovitz A, Goldsher D. Ischemic stroke due to a calcified embolus from the mitral annular valve. Neurology. 2012;78:931

6. Deng $Q$, Zhang Y, Ding H, Dong Q, Fu J. Calcific emboli originating from the brachiocephalic trunk causing acute cerebral infarction and worm-like calcification in the right middle cerebral artery. Journal of clinical neuroscience : official journal of the Neurosurgical Society of Australasia. 2015;22:889-890

7. Konishi-Yakushiji M, Yakushiji Y, Kotooka N, Okajima Y, Noguchi T, Hara H. Sonographic confirmation of the association between calcified cerebral emboli and mitral annular calcification. Journal of ultrasound in medicine : official journal of the American Institute of Ultrasound in Medicine. 2010;29:1507-1510

8. Tardy J, Da Silva N, Glock Y, Larrue V. Neurological pictures. Stroke with calcium emboli related to a calcified stenosis of internal carotid artery. Journal of neurology, neurosurgery, and psychiatry. 2008;79:1273-1274

9. Chandran V, Pai A, Rao S. Calcified embolism: A rare cause of cerebral infarction. $B M J$ case reports. 2013;2013

10. Weinberger J, Marks SJ, Gaul JJ, Goldman B, Schanzer H, Jacobson J, et al. Atherosclerotic plaque at the carotid artery bifurcation. Correlation of ultrasonographic imaging with morphology. Journal of ultrasound in medicine : official journal of the American Institute of Ultrasound in Medicine. 1987;6:363-366

11. Weinberger J, Ramos L, Ambrose JA, Fuster V. Morphologic and dynamic changes of atherosclerotic plaque at the carotid artery bifurcation: Sequential imaging by real time b-mode ultrasonography. Journal of the American College of Cardiology. 1988;12:1515-1521

12. Weinberger J, Robbins A. Neurologic symptoms associated with nonobstructive plaque at carotid bifurcation. Analysis by real-time b-mode ultrasonography. Arch Neurol. 1983;40:489492

13. Cerase A, Grazzini I. Early relapsing calcified cerebral embolism. J Stroke Cerebrovasc Dis. 2015;24:e125-126

14. Fisher M, Paganini-Hill A, Martin A, Cosgrove M, Toole JF, Barnett HJ, et al. Carotid plaque pathology: Thrombosis, ulceration, and stroke pathogenesis. Stroke; a journal of cerebral circulation. 2005;36:253-257

15. Topakian R, King A, Kwon SU, Schaafsma A, Shipley M, Markus HS. Ultrasonic plaque echolucency and emboli signals predict stroke in asymptomatic carotid stenosis. Neurology. 2011;77:751-758 